\title{
Large paracetamol overdose - higher dose NAC is required
}

\author{
D Bateman ${ }^{1}$ \\ ${ }^{1}$ University of Edinburgh Division of Clinical and Surgical Sciences
}

July 13, 2021

\begin{abstract}
Paracetamol poisoning continues to be a worldwide problem and despite the availability of an affective antidote, N-acetylcysteine (NAC), the optimal way to use this antidote, particularly following very large doses of paracetamol, has not been established. Recent case series have shown an increased toxicity from paracetamol, even in those receiving prompt NAC therapy, at high doses of paracetamol, particularly in patients above the $300 \mathrm{mg} / \mathrm{L}$ nomogram treatment line. Clinical trial evidence supporting shorter NAC dosing now allows the possibility for intensifying treatment without the risk of very high rates of ADRs. New biomarkers also show the possibility of early identification of patients at risk of liver injury who might also benefit from increased intensity treatment. This article discusses these data and proposes a logical therapy for increasing NAC dosing which now requires clinical trial testing.
\end{abstract}

Large paracetamol overdose - higher dose NAC is required

What is already known

Paracetamol poisoning is the commonest acute poisoning presentation in the UK and frequent worldwide. It causes a well-recognised clinical syndrome, resulting in death from liver failure in the most severe cases. Risk of death is greater in larger overdoses and later hospital presentation.

In the 1970's a treatment for paracetamol poisoning was developed, intravenous $\mathrm{N}$ acetylcysteine (NAC). This was enormously effective, but the first dose used in published studies, $300 \mathrm{mg} / \mathrm{kg}$ body weight is still used for every case of paracetamol poisoning, whatever the dose.

Some authors have recently advocated more intense use of NAC in larger paracetamol overdoses.

Novel methods of delivering IV NAC that reduce ADR rates facilitate this approach.

What this article adds

An argument for a focussed use of more intensive NAC in large paracetamol overdose is presented.

A method for giving such higher doses is discussed.

Formal studies of this approach are proposed, and their challenges discussed.

Abstract

Paracetamol poisoning continues to be a worldwide problem and despite the availability of an affective antidote, N-acetylcysteine (NAC), the optimal way to use this antidote, particularly following very large doses of paracetamol, has not been established. Recent case series have shown an increased toxicity from paracetamol, even in those receiving prompt NAC therapy, at 
high doses of paracetamol, particularly in patients above the $300 \mathrm{mg} / \mathrm{L}$ nomogram treatment line.

Clinical trial evidence supporting shorter NAC dosing now allows the possibility for intensifying treatment without the risk of very high rates of ADRs. New biomarkers also show the possibility of early identification of patients at risk of liver injury who might also benefit from increased intensity treatment. This article discusses these data and proposes a logical therapy for increasing NAC dosing which now requires clinical trial testing.

\section{Introduction}

It is hard to imagine a clinical pharmacologist today approaching the dose an antidote based on a strategy of the same dose for every case. Nevertheless, for paracetamol (acetaminophen) this approach has been successful has been used for almost 50 years, and there is little doubt has saved many lives. Is it now time to reassess this 'one dose for all' policy?

\section{Historical background}

The management of paracetamol poisoning was changed fundamentally in the 1970s by the discovery of the mechanism of toxicity of paracetamol, metabolic transfer to a reactive metabolite N-acetyl benzoquinoneimine (NABQI), and depletion of hepatic cysteine as the cause of toxicity. ${ }^{1}$ Studies in animals and man clearly showed a dose response relationship between paracetamol dose, hepatotoxicity, risk of liver failure and death. Treatment with sulfhydryl donors was shown to be very effective in preventing this process in animal models, with good evidence for the time criticality of treatment but weaker evidence of a dose response relationship to the effects of sulfhydryl donors such as cysteamine. ${ }^{1,2}$ Prescott first showed the efficacy of NAC in a series of cases of paracetamol overdose in Edinburgh. ${ }^{3}$

At that time the science of clinical trials was it in its infancy and dose ranging studies in man of NAC were not done. The first dose used in the UK IV acetylcysteine (NAC) proved highly effective and soon became the antidote of choice. The dose used $(300 \mathrm{mg} \mathrm{NAC} / \mathrm{kg}$ ) was intravenous given over 20.5 hours in three aliquots, $150 \mathrm{mg} / \mathrm{kg}$ over $15 \mathrm{~min}, 50 \mathrm{mg} / \mathrm{kg}$ over $4 \mathrm{~h}$ and $100 \mathrm{mg} / \mathrm{g}$ over $20 \mathrm{~h}^{2,4}$ An alternative 3 day, 4 hourly dose, oral regimen was developed in the United States following discussions between Rumack and the FDA. ${ }^{5,6}$ In both cases the total dose of antidote was based empirically on calculations using estimates of the rates of production of the toxic metabolite of paracetamol, NABQI. ${ }^{6}$ Once an effective dose was decided in the 1970's it was deemed unethical to conduct further clinical trials on IV NAC dose in the UK.

Since the original case series of untreated cases in the early 1970's the use of NAC has been determined by paracetamol dose, optimally assessed by plasma concentration, ideally 4-12 hours after ingestion, although intervention doses and concentrations vary worldwide with implications for numbers of patients treated. ${ }^{7}$ Toxicity prediction is more difficult following multiple ingestions or in delayed presentation.

IV NAC causes adverse reactions in a significant proportion of patients, their frequency over $60 \%$ for vomiting and $30 \%$ for anaphylactoid reactions in clinical trial conditions, are related to the initial dosing rate. We showed they can be significantly reduced by a modified NAC regimen as shown in the SNAP trial that used $100 \mathrm{mg}$ IV over $2 \mathrm{~h}$ and $200 \mathrm{mg} / \mathrm{kg}$ over $10 \mathrm{~h} .{ }^{8,9}$ Additionally such reactions are more common in patients with lower concentrations of paracetamol, and although very rarely fatal, they are nevertheless problematic in clinical practice as they require interruption to NAC treatment. Despite these advances the standard intravenous dose of NAC has remained based on the patient's weight, rather than the dose of paracetamol.

Since NAC was introduced there has been discussion on which groups of patients should receive it. Policies across the world still vary from the extreme 'treat everybody' in Denmark to a more structured framework operated everywhere else based on a combination of plasma concentration of paracetamol, stated ingested dose and timeframe, time from ingestion to presentation and blood tests taken to assess liver function and clotting factors both to determine need for initial therapy in late presentations and to decide whether to continue treatment in patients developing liver injury. ${ }^{10}$ This article will therefore focus on exploring what 
evidence there is to change this approach in the face of high paracetamol overdoses, and at what points in therapy intensification of NAC therapy might be considered and put into practice.

\section{Methods}

In approaching this topic, a search of PubMed was done using index terms: paracetamol; acetylcysteine; dose; toxicity; massive paracetamol overdose.

Of the many thousand references identified the author has manually chosen those that specifically deal with the areas to be covered in this discussion and identified references also using a large personal collection that are relevant.

A focus on the 3 areas most apposite to the issue of increasing doses of NAC in large paracetamol overdose has been adopted, these being evidence that:-

1. larger doses of paracetamol cause increased toxicity even with NAC therapy

2. increasing NAC dose may have efficacy in large paracetamol overdoses

3. the various modes of action suggested for NAC that may be relevant to any increased efficacy from a larger NAC dose.

\section{Results}

\section{Larger doses of paracetamol}

Rumack and Bateman reviewed the then chosen doses of NAC in $2012^{6}$. They discuss in detail how in the 1970's work in animals and man was used to calculate the amount of the mercapturate conjugate of paracetamol, the end point of NAPQI metabolism before cysteine depletion and hence determine an estimated effective dose of NAC for human poisoning based on Mitchell et al who had suggested that a dose of $15 \mathrm{~g}$ of paracetamol was the toxic threshold. ${ }^{11}$ Rumack and Bateman used these data to establish likely NAC requirements (dose $/ \mathrm{kg} / \mathrm{h}$ ) in a $70 \mathrm{~kg}$ patient ingesting from 15.9 to $47.7 \mathrm{~g}$ paracetamol, these doses being chosen to simplify the calculations. At the highest dose they estimated the NAC requirement to be a $17.75 \mathrm{mg} / \mathrm{kg} / \mathrm{h}$. Their calculations are untested in patients, and subject to potential error. ${ }^{6}$ Thus at this concentration a $70 \mathrm{~kg}$ patient with a VD of approximately $0.9 \mathrm{~L} / \mathrm{kg}$ would have $18.9 \mathrm{~g}$ of paracetamol in their body $4 \mathrm{~h}$ after their initial dose. As the bioavailability of paracetamol at therapeutic dose is about $75 \%$ and half-life in untreated patients of the order of $2 \mathrm{~h}$ it is clear that a dose well above $20 \mathrm{~g}$ would be necessary to achieve these concentrations. ${ }^{12} \mathrm{~A}$ problem with such calculations is that clearance of paracetamol is impaired in overdose, but as the half-life in non-toxic patients is of the order of $2 \mathrm{~h}$, it can be seen that suggestions of $50 \mathrm{~g}$ as a cut off for "massive overdose" may have some basis in theory.

Supportive evidence for inadequacy of current NAC doses in large overdose that this has

been found in 2 UK case series $\left({ }^{13,14}\right)$ and one from Australia. $\left({ }^{15}\right)$ All three showed a dose dependant increase in toxicity with a clear upturn in risk with a nomogram concentration above the $300 \mathrm{mg} / \mathrm{L}$ nomogram line $(300 \mathrm{mg} / \mathrm{L}$ at $4 \mathrm{~h}$ post OD; line half-life $4 \mathrm{~h})$ in those treated within $8 \mathrm{~h}$ in the $2 \mathrm{UK}$ studies.

Increasing NAC dose efficacy in large paracetamol overdoses

The term 'massive overdose' has been used by some as a term to cover this kind of patient, but we currently lack hard clinical trial evidence on this approach. While there are case reports using increased NAC doses to date there has been a lack of a clear research strategy. These cases are rare and will therefore require multicentre studies, making expense a significant barrier to clinical trials under current UK regulations. There is nevertheless clear theoretical base for such an approach as discussed above.

The if the patient presents with a large overdose, and presentation is delayed beyond 8-12 h, making the need to treat more urgent a dose corrected for body weight of

$500 \mathrm{mg} / \mathrm{kg}$ body weight seems an alternative simple cut-off for considering a higher, doubled intensity treatment. ${ }^{16}$ This has the advantage of simplicity as opposed to more complex algorithms with varying 
NAC doses across a range of higher

paracetamol doses. ${ }^{6,17}$

Modes of action of NAC

The mechanisms of paracetamol-induced hepatic damage are now understood to be more complex than just cysteine depletion, and a series of other targets in the process of liver injury have been suggested for therapy, particularly for mitochondrial injury, the response and process of necrotic hepatocyte death including cell signalling processes, tumour suppressor proteins that modify the cell cycle (p53) and nuclear factor erythroid 2-related factor 2 (Nrf2) and as a hydrogen sulfide precursor. ${ }^{18-21}$ Furthermore NAC might exert therapeutic effects in other organs rather than just as a sulfhydryl donor ${ }^{22,23}$. If relevant to the liver in human acute paracetamol induced liver failure these mechanisms would help to explain benefit of NAC in later stages of paracetamol-induced hepatic failure. ${ }^{24,25}$

\section{Discussion}

Cases of very large paracetamol overdoses are unusual, but these patients present specific

problems for management. There is clearly a sound theoretical basis for using an

increased dose of NAC as soon as practicable in the management of such patients.(Rumack

and Bateman 2012) Other treatments have been suggested, including haemodialysis, for

which there is reasonable evidence of efficacy in removal of paracetamol, but much less of

hepato-protection. ${ }^{26,27}$ Similarly metabolic antidotes, including fomepizole and calmangafodipir, which are both in clinical trial and have been studied on a case basis. ${ }^{17,28-30}$

Identifying patients at increased risk may be difficult as there is a variability in the

susceptibility to paracetamol which is still not fully understood. ${ }^{31}$ Obviously very high doses present a risk, but newer molecular techniques now allow identification of patients at risk of liver injury at hospital presentation. ${ }^{32-34}$ As these are introduced into therapy routinely the management of this common poisoning should change again. One significant advance has happened the use of a modified NAC dosing regimen (SNAP) first described in 2014 which now has both clinical trial evidence and reasonable outcome data..$^{8,35}$ This regimen uses a 12-hour tailored infusion of $300 \mathrm{mg}$ per kilogram NAC. It therefore delivers the current international standard dose but over a shorter period. It effectively intensifies early overdose management but with far fewer adverse effects. This offers opportunities to further intensify NAC therapy.

The first opportunity is at presentation, if there is a clear history of large overdose, when doubling of the infusion should provide reasonable protection for the vast majority of high quantity overdoses, if administered within the first 12 hours. A second opportunity for continuing this higher dose, or increasing a standard dose occurs at the end of this 12 hour period. At present in this regimen bloods are taken 10 hours into the infusion in order that clear therapeutic decisions can be made prior to the end of the infusion. Thus, even if a standard dose of $300 \mathrm{mg}$ per kilogram is used initially this can be repeated, or even doubled, if blood test suggest liver damage is occurring, or paracetamol clearance is significantly delayed. This is in contrast to standard regimens which, allow intensification at presentation or at $21 \mathrm{~h}$ and with dose doubling of this will therefore deliver a smaller dose in this first 21 hours than intensifying the SNAP regimen.

This intensified regimen has not been subject to clinical trial, and it seems unlikely that it would be possible to do this within the present environment of the UK and the numbers of very high ingestions seen. Most likely an international collaborative study with you would be required. The present evidence base is however far stronger for this approach than for any other currently being proposed.

\section{Conclusion}


The time has come to use a higher dose of NAC in patients presenting with high-dose overdose, over $500 \mathrm{mg} / \mathrm{kg}$ body weight, or with evidence of developing hepatic damage while a standard $12 \mathrm{~h}$ does of NAC is being used. The animal and human data show that the earlier NAC is used the better the outcome, and it would be illogical not to assume the same in larger paracetamol overdose, further justifying an earlier intensification of NAC and supporting early assessment of response that is facilitated by the shorter SNAP NAC regimen. ${ }^{8}$

Data sharing statement.

No research data are shared in this article

\section{References}

1. Mitchell JR, Thorgeirsson SS, Potter WZ, Jollow DJ, Keiser H. Acetaminophen-induced hepatic injury: Protective role of glutathione in man and rationale for therapy. Clin Pharm Ther . 1974;4:676-684.

2. Prescott LF, Park J, Ballantyne A, et al. Treatment of paracetamol (acetaminophen) poisoning with N-acetylcysteine. . Lancet . 1977;2(8035):432-434.

3. Prescott LF, Sutherland GR, Park J, Smith IJ, Proudfoot AT. Cysteamine, methionine, and penicillamine in the treatment of paracetamol poisoning. Lancet . 1976;2(7977):109-113.

4. Prescott LF, Illingworth RN, Critchley JA, Stewart MJ, Adam RD, Proudfoot AT. Intravenous Nacetylcystine: the treatment of choice for paracetamol poisoning. Br Med J . 1979;2(6198):1097-1100.

5. Smilkstein MJ, Knapp GL, Kulig KW, Rumack BH. Efficacy of oral N-acetylcysteine in the treatment of acetaminophen overdose. Analysis of the national multicenter study (1976 to 1985). N Engl J Med . 1988;319(24):1557-1562. doi:10.1056/NEJM198812153192401

6. Rumack BH, Bateman DN. Acetaminophen and acetylcysteine dose and duration: Past, present and future. Clin Toxicol . 2012;50(2):91-98. doi:10.3109/15563650.2012.659252

7. Bateman DN, Dear JW. Acetylcysteine in paracetamol poisoning: A perspective of 45 years of use. Toxicol Res (Camb) . 2019;8(4):489-498. doi:10.1039/c9tx00002j

8. Bateman DN, Dear JW, Thanacoody HKR, et al. Reduction of adverse effects from intravenous acetylcysteine treatment for paracetamol poisoning: A randomised controlled trial. Lancet . 2014;383(9918):697-704. doi:10.1016/S0140-6736(13)62062-0

9. Chiew A, Gluud C, Brok J, Buckley N. Interventions for paracetamol ( acetaminophen ) overdose. Cochrane Database Syst Rev . 2018;(2):1-90. doi:10.1002/14651858.CD003328.pub3.www.cochranelibrary.com

10. Ferner RE, Dear JW, Bateman DN. Management of paracetamol poisoning.Bmj . 2011;342(7804):968972. doi: $10.1136 /$ bmj.d2218

11. Mitchell JR, Thorgeirsson SS, Potter WZ, Jollow DJ, Keiser H. Acetaminophen-induced hepatic injury: protective role of glutathione in man and rationale for therapy. Clin Pharmacol Ther . 1974;16(4):676-684.

12. Forrest JA, Clements JA, Prescott LF. Clinical pharmacokinetics of paracetamol. Clin Pharmacokinet . 1982;7(2):93-107.

13. Cairney DG, Beckwith HKS, Al-Hourani K, Eddleston M, Bateman DN, Dear JW. Plasma paracetamol concentration at hospital presentation has a dose-dependent relationship with liver injury despite prompt treatment with intravenous acetylcysteine. Clin Toxicol . 2016;54:405-410.

14. Marks DJ, Dargan PI, Archer JR, et al. Outcomes from massive paracetamol overdose: a retrospective observational study. Br J Clin Pharmacol . 2017;(83):1263-1272.

15. Chiew AL, Isbister GK, Duffull SB, Buckley NA. Evidence for the changing regimens of acetylcysteine. Br J Clin Pharmacol . 2016;81(3):471-481. doi:10.1111/bcp.12789 
16. Bateman DN, Dear JW. Should we treat very large paracetamol overdose differently? $\mathrm{Br} J$ Clin Pharmacol . 2017;83(6):1163-1165. doi:10.1111/bcp.13279

17. Hendrickson RG. What is the most appropriate dose of $\mathrm{N}$-acetylcysteine after massive acetaminophen overdose? Clin Toxicol . 2019;57(0):686-691. doi:10.1080/15563650.2019.1579914

18. Yan M, Huo Y, Yin S, Hu H. Mechanisms of acetaminophen-induced liver injury and its implications for therapeutic interventions. Redox Biol . 2018;17(April):274-283. doi:10.1016/j.redox.2018.04.019

19. Ramachandran A, Jaeschke H. Mechanisms of acetaminophen hepatotoxicity and their translation to the human pathophysiology.J Clin Transl Res . 2017;3:157-169. doi:10.18053/jctres.03.2017s1.002

20. Jaeschke H, Ramachandran A. Acetaminophen Toxicity: Novel Insights Into Mechanisms and Future Perspectives. Gene Expr . 2018;18:19-30. doi:10.3727/105221617X15084371374138

21. Ezeringa D, Takano Y, Hanaoka K, Urano Y, Dick TP. N-Acetyl Cysteine Functions as a Fast-Acting Antioxidant by Triggering Intracellular H 2 S and Sulfane Sulfur Production. Cell Chem Biol . 2018;25(4):447459.e4. doi:10.1016/j.chembiol.2018.01.011

22. Rushworth GF, Megson IL. Existing and potential therapeutic uses for N-acetylcysteine: The need for conversion to intracellular glutathione for antioxidant benefits. Pharmacol Ther . 2014;141(2):150-159. doi:10.1016/j.pharmthera.2013.09.006

23. Karuppagounder SS, Alin L, Chen Y, et al. N-acetylcysteine targets 5 lipoxygenase-derived, toxic lipids and can synergize with prostaglandin E2 to inhibit ferroptosis and improve outcomes following hemorrhagic stroke in mice. Ann Neurol . 2018;84(6):854-872. doi:10.1002/ana.25356

24. Keays R, Harrison PM, Wendon JA, et al. Intravenous acetylcysteine in paracetamol induced fulminant hepatic failure: a prospective controlled trial. Bmj . 1991;303(6809):1026-1029.

25. Harrison PM, Keays R, Bray GP, Alexander GJ, Williams R. Improved outcome of paracetamol-induced fulminant hepatic failure by late administration of acetylcysteine. Lancet . 1990;335(8705):1572-1573.

26. Ghannoum M, Kazim S, Grunbaum AM, Villeneuve E, Gosselin S. Massive acetaminophen overdose: effect of hemodialysis on acetaminophen and acetylcysteine kinetics. Clin Toxicol (Phila) . 2016;54(6):519522. doi:10.1080/15563650.2016.1175006

27. Gosselin S, Juurlink DN, Kielstein JT, et al. Extracorporeal treatment for acetaminophen poisoning: recommendations from the EXTRIP workgroup. Clin Toxicol . 2014;52(8):856-867. doi:10.3109/15563650.2014.946994

28. Woolum JA, Hays WB, Patel KH. Use of fomepizole, n-acetylcysteine, and hemodialysis for massive acetaminophen overdose. Am J Emerg Med . 2020;38(3):692.e5-692.e7. doi:10.1016/j.ajem.2019.09.026

29. Morrison EE, Oatey K, Gallagher B, et al. Principal results of a randomised open label exploratory, safety and tolerability study with calmangafodipir in patients treated with a $12 \mathrm{~h}$ regimen of $\mathrm{N}$-acetylcysteine for paracetamol overdose (POP trial).EBioMedicine . 2019;46:423-430. doi:10.1016/j.ebiom.2019.07.013

30. Mullins ME, Yeager LH, Freeman WE. Metabolic and mitochondrial treatments for severe paracetamol poisoning: a systematic review.Clin Toxicol . 2020;58(12):1284-1296. doi:10.1080/15563650.2020.1798979

31. Caparrotta TM, Antoine DJ, Dear JW. Are some people at increased risk of paracetamol-induced liver injury? A critical review of the literature. Eur J Clin Pharmacol . 2018;74(2):147-160. doi:10.1007/s00228017-2356-6

32. Vliegenthart AD, Kimmitt RA, Seymour JH, et al. Circulating acetaminophen metabolites are toxicokinetic biomarkers of acute liver injury. Clin Pharmacol Ther . 2016;10. doi:10.1002/cpt.541

33. Dear JW. New Biomarkers for Drug-Induced Liver Injury.Hepatology . 2018;67(6):2479-2480. doi:10.1002/hep. 29825 
34. Nuzzo A, Salem S, Malissin I, et al. in acetaminophen poisoning : A prospective cohort study. $U E G$ J . 2021;9:571-580. doi:10.1002/ueg2.12093

35. Pettie JM, Caparrotta TM, Hunter RW, et al. Safety and Efficacy of the SNAP 12-hour Acetylcysteine Regimen for the Treatment of Paracetamol Overdose. EClinicalMedicine . 2019;11:11-17. doi:10.1016/j.eclinm.2019.04.005 